\title{
Azathioprine for The Induction Treatment of Connective Tissue Disorder Related Interstitial Lung Disease and Comparison with Cyclophosphamide: a retrospective cohort analysis
}

\author{
Hasan Satış${ }^{1}$, Mehmet Onut ${ }^{2}$, Caner Baysan ${ }^{3}$, Reyhan Bilici Salman ${ }^{1}$, Dilek Yapar ${ }^{1}$, Hakan \\ Babaoğlu ${ }^{1}$, Nuh Ataş ${ }^{1}$, Aslıhan Avanoğlu Güler ${ }^{1}$, Hazan Karadeniz ${ }^{1}$, Hamit Küçük ${ }^{1}$, \\ Seminur Haznedaroglu ${ }^{4}$, BURCU GOKER ${ }^{4}$, Mehmet Akif Öztürk ${ }^{1}$, Abdurrahman Tufan ${ }^{4}$, \\ Nilgün Yllmaz Demirci ${ }^{1}$, and Haluk Türktaş ${ }^{1}$ \\ ${ }^{1}$ Gazi University Faculty of Medicine \\ ${ }^{2}$ Ankara State Hospital \\ ${ }^{3}$ Ankara University \\ ${ }^{4}$ Gazi University
}

April 9, 2021

\begin{abstract}
Objectives: Treatment of connective tissue disease-related interstitial lung disease (CTD-ILD) remains challenging. The literature related to the immunosuppressive drugs is very limited in most CTDs and there is no previously reported study comparing induction regimens in patients with newly defined 'interstitial pneumonia with autoimmune features (IPAF), We aimed to investigate the efficacy of azathioprine (AZA) used in induction regimens for interstitial lung disease in a variety of connective tissue disorders including IPAF, and compare it with cyclophosphamide (CYC) Methods. In a retrospective study, all patients presented with interstitial lung disease to the rheumatology and/or pulmonology departments in a tertiary referral centre, between 2009 and 2019. Five major CTD groups were defined; systemic sclerosis, rheumatoid arthritis (RA), primary Sjögren syndrome (pSS), inflammatory myositis (IMS) and interstitial pneumonia with autoimmune features (IPAF). All patients who satisfied one of these and received AZA or CYC for induction therapy for at least 6 months were included in the study. Treatment responses at six months and side effects were analyzed. Results: There were 1351 patients diagnosed with ILD. Of these, 328 patients were identified as CTD-ILD, satisfying the classification criteria of one of the CTDs. Among these, 57 patients received AZA and 79 patients received CYC for induction therapy. CYC treatment resulted in a $2.41 \%$ increase in FVC, however, AZA resulted in a $1.44 \%$ decline in FVC predicted $(\mathrm{p}=0.041)$. Propensity score matching was used to reduce selection bias, AZA treatment was related to increased risk of progression (50\% vs 13.5\%, p:0.002). Conclusion: CYC is superior to AZA in induction therapy of CTD-ILD.
\end{abstract}

Azathioprine for The Induction Treatment of Connective Tissue Disorder Related Interstitial Lung Disease and Comparison with Cyclophosphamide: a retrospective cohort analysis

\section{Abstract}

Objectives: Treatment of connective tissue disease related interstitial lung disease (CTD-ILD) remains challenging. The literature related to the immunosuppressive drugs is very limited in most CTDs and there is no previously reported study comparing induction regimens in patients with newly defined 'interstitial pneumonia with autoimmune features (IPAF), We aimed to investigate the efficacy of azathioprine (AZA) used in induction regimens for interstitial lung disease in a variety of connective tissue disorders including IPAF, and compare it with cyclophosphamide (CYC) 
Methods. In a retrospective study, all patients presented with interstitial lung disease to the rheumatology and/or pulmonology departments in a tertiary referral center, between 2009 and 2019. Five major CTD groups were defined; systemic sclerosis, rheumatoid arthritis (RA), primary Sjögren syndrome (pSS), inflammatory myositis (IMS) and interstitial pneumonia with autoimmune features (IPAF). All patients who satisfied one of these and received AZA or CYC for induction therapy for at least 6 months were included in the study. Treatment responses at six months and side effects were analyzed.

Results : There were 1351 patients diagnosed with ILD. Of these, 328 patients were identified as CTDILD, satisfying classification criteria of one of the CTDs. Among these, 57 patients received AZA and 79 patients received CYC for induction therapy. CYC treatment resulted in $2.41 \%$ increase in FVC, however AZA resulted in $1.44 \%$ decline in FVC predicted $(\mathrm{p}=0.041)$. Propensity score matching was used to reduce selection bias, AZA treatment was related to increased risk of progression ( $50 \%$ vs $13.5 \%$, p:0.002) .

Conclusion: CYC is superior to AZA in induction therapy of CTD-ILD.

Key words: Azathioprine, Connective tissue disease, Cyclophosphamide, Interstitial lung disease, , interstitial pneumonia with autoimmune features, treatment

\section{What's already known about this topic?}

Interstitial lung disease (ILD) is one of the leading cause of mortality and morbidity for connective tissue disease. Immunosuppressive drugs are the main treatment for ILD. Cyclophosphamide (CYC) and azathioprine (AZA) are the leading treatment options for CTD-ILD and data related to those treatments mostly come from studies on scleroderma patients. Head to head comparison of CYC and AZA in first line treatment of CTD-ILD is also lacking.

\section{What does this article add?}

CYC is a better option for the first line treatment of ILD in systemic sclerosis, rheumatoid arthritis and primary sjögren syndrome patients compared to AZA treatment. AZA could be an alternative option in inflammatory myositits associated ILD and interstitial pneumonia with autoimmune features with limited disease extent .

\section{Introduction}

Interstitial lung disease (ILD) is characterized by replacement of normal pulmonary parenchyma with inflammatory cells and/or fibrotic tissues which results in progressive loss of functional lung units that impairs gas exchange. Connective tissue diseases (CTDs) are one of the main causes of ILD and lung involvement is the leading morbidity and mortality reason in connective tissue disorders [1, 2]. Although ILD is most commonly observed in systemic sclerosis [3] and rheumatoid arthritis (RA) it can be seen in nearly all CTDs[4].Due to high mortality and morbidity of CTD-ILD, selecting optimal immunosuppressive regimen considering type and severity of underlying rheumatic disease, progression of pulmonary impairment and drug tolerance/side effects.

Immunosuppressive drugs remain as the main stay of therapy in CTD-ILD. Azathioprine (AZA) and cyclophosphamide (CYC) are among the most commonly employed immunosuppressive drugs. AZA inhibits both $\mathrm{T}$ and $\mathrm{B}$ cell proliferation[5], whereas CYC, an alkylating agent, inhibits T cell proliferation by inducing its apoptosis and decreases both humoral and cellular immune responses[6, 7]. There are several studies regarding use of CYC as induction regimen in CTD-ILD, which showed clear benefit in stabilization and improvement in lung functions $[8,9]$. Although CYC is effective in preserving lung function, it loses its efficacy after discontinuation and necessitates long term treatment, which is significantly limited by its cumulative toxicity. Therefore, CYC is mostly used in induction regimens, followed by maintenance therapy with other drugs, such as AZA or mycophenolate mofetil (MMF).In a retrospective study, AZA was compared with MMF and found to have similar safety and efficacy for stabilization of lung functions [10].

Despite its favorable toxicity profile compared to CYC, studies in which AZA is used in induction regimens are quite scarce. There were two studies which compared efficacy of AZA and CYC in induction treatment of 
systemic sclerosis [3] related ILD, both reported stabilization of lung functions with AZA[11, 12]. A case series of 11 patients with primary Sjögren's syndrome (pSS) related ILD, authors reported favorable responses with AZA based induction regimen, but lack of a comparator drug limited implementation of results [13]. To the best of our knowledge, AZA based regimens in ILD patients with interstitial pneumonia with autoimmune features (IPAF) has not been previously reported.

Therefore, studies on efficacy of AZA in CTD-ILD are still needed, especially for those with IPAF or RA, and also in other cases in which CYC is contraindicated or not tolerated. Herein, we retrospectively investigated efficacy and safety of AZA in the treatment of ILD and compared it with intravenous ( iv) CYC in a relatively large cohort.

\section{Methods}

Computer based medical records of our ILD cohort were investigated. ILD diagnosis was confirmed by evaluation of high resolution computed tomography (HRCT) images. Diagnoses of CTDs (SSc, RA, pSS, DM/PM, IPAF) were made with their corresponding classification criteria [14-18]. Demographic features, smoking history, type of symptoms, time at onset of respiratory symptoms, time at diagnosis, laboratory results, serologic tests including anti-nucleolar antibodies, extractable nuclear antigen (ENA) panel, rheumatoid factor, anti-citrullinated peptide and anti-neutrophil cytoplasmic antibodies were recorded.

Our ILD cohort was established in 2010 jointly by members from rheumatology and pulmonary departments. Since then, team members meet weekly to evaluate CTD-ILD patients (including symptoms, physiologic tests and imaging studies) and make decisions for individual treatment plans. Functional assessment of patients were performed by Modified Medical Research Council (MMRC)[19]. Pulmonary function tests (PFTs) were performed at baseline and every 6-months periods thereafter including; forced expiratory volume in 1 min (FEV1), forced vital capacity (FVC), and DLCO, expressed as percentages of predicted values adjusted fora ge, sex, height and hemoglobin level, according to techniques accepted by the American Thoracic Society (ATS)[20]. HRCT was performed at baseline then annually, or in case of a new significant respiratory symptom that suggest acute exacerbation of lung disease or significant deterioration of physiological parameters. ILD patterns were classified as non-specific interstitial pneumonia (NSIP), usual interstitial pneumonia (UIP) according to HRCT findings including ground glass opacities, traction bronchiectasis and sub-pleural honeycombing. Pulmonary involvement in HRCT in SSc patients was graded as limited or extended based on extent of reticular pattern, as suggested per consensus of experts[21]. In this consensus report, experts suggest to evaluate HRCT at 5 different levels for total disease extent of reticulation, for other rheumatologic disease ILD disease there is not any other radiological scoring system, thus same scoring system was applied for those sub-types. Modified Gender-Age-Physiology [22] index was used for prognosis [23].

Medications used, including systemic corticosteroids, synthetic and biologic disease-modifying anti-rheumatic drugs (DMARD), AZA, CYC and were meticulously reviewed. There was not any other immunsuppresive drugs for the treatment of ILD during the induction therapy, which took six months. Clinically significant side effects were nausea/vomiting/abdominal pain impairing oral intake, increased liver function tests[?]3 timesof upper limit of normal, neutrophil count of $<1500 / \mathrm{mm}^{3}$, thrombocyte count $<150,000 / \mathrm{mm}^{3}$, and hemorrhagic cystitis.

Primary outcome measure was treatment response at six-month after introduction of induction therapy. Radiological progression was defined as, more than $10 \%$ increase in reticular pattern on HRCT, functional progression defined as worsening in FVC greater than 10\% or DLCO greater than 15\%and clinical progression defined as increase in MMRC score. The patients had to have detoration in at least two of these domains in order to have progressive disease.

Categorical variables were given as numbers and percentages. Continuous data were as mean +- standard deviation (SD) or median (interquartile range). Conformity of continuous variables to normal distribution was evaluated using visual (histogram and probability graphs) and analytical methods (Kolmogorov-Smirnov / Shapiro-Wilk tests). If variables were normally distributed Student t-test was used, if not, Mann Whitney $\mathrm{U}$ test was used for comparison. Comparison of categorical variables was made with Chi-square or Fisher's 
exact tests. A p-value of 0.05 or less was considered as statistically significant in all analyzes. Propensity score was used to explain the differences of patient groups receiving AZA and CYC. The propensity score value for each patient was calculated using a logistic regression model containing the determined variables. Two to one (One to two (1:2)) nearest-neighbor matching and caliper width of 0.2 (caliper width of 0.2 ) were performed.

\section{RESULTS}

ILD cohort consisted of 1351 patients and 314 of them were identified as CTD associated ILD, satisfying classification criteria of one of the CTDs. Patients followed up less than 6 months were excluded. Among the remaining, 57 patients received AZA and and 79 patients were given CYC for induction therapy and were included.

Demographics and disease characteristics of the study patients are presented in Table 1. AZA treated patients were significantly older, had shorter duration of pulmonary symptoms, less extensive disease and better forced vital capacity (FVC) at baseline compared to CYC group (Table 1). NSIP pattern was more frequently seen in all sub-groups except for RA patients. Patients diagnosed with newly defined IPAF were significantly more likely to get AZA, whereas, those with SSc were more likely to get CYC for induction therapy.

\section{Treatment Efficacy}

In AZA group, 10 patients discontinued their treatment before 6 months and four patients had incomplete data, leaving 43 patients for the final analysis. Seven patients in CYC group had missing data and 72 patients were included in the final analysis. Treatment outcomes are summarized in Table 2. Despite favorable baseline parameters, those who received AZA for induction therapy were significantly more likely to progress in 6 months, in terms of MMRC scores and regression of intensity of reticulation on HRCT, as well as \% predicted FVC. CYC treatment resulted in $2.41 \%$ increase in FVC, however AZA resulted in $1.44 \%$ decline in FVC predicted $(\mathrm{p}=0.041)$. These findings remained consistent after adjustment for age, gender and HRCT pattern, CTD diagnosis, smoking status, corticosteroid use, disease extent and duration of symptoms. Progression was more frequent in AZA group across all disease subtypes. After adjusting for potential confounding factors (age, disease subtype and basal FVC), a multivariate regression model was created. The analysis showed that CYC treatment was associated with decreased risk of progression compared to AZA treatment (HR: 0.18 (95\% CI 0.05 to 0.542)). Propensity score was used to explain the differences between patient groups receiving AZA and CYC. The variables used in the calculation for patients are: age, gender, smoking status, pre-treatment FVC percent and disease subtype (SCL / nonSCL). According to the propensity score matching status, a total of 65 patients, $28 \mathrm{AZA}$ and $37 \mathrm{CYC}$, were selected.After this calculation AZA treatment was related to increased risk of progression compared to CYC treatment (HR: 6.75 (95 CI \%, 1.97 to 23.12))

\section{Safety Analysis}

In general, treatment modalities were both well tolerated. $88.3 \%$ in AZA group and $90.4 \%$ in CYC group completed six months induction therapy. Major side effects are shown in Table 3. In AZA group, 10 patients had side effects that resulted in treatment discontinuation in the first week of treatment. Four patients had severe nausea-vomiting, two patients abdominal pain/bloating, four patients had liver function abnormalities and one patient developed thrombocytopenia and neutropenia. In five patients, dosage reduction was needed in AZA, two due to elevated liver enzymes and three due to gastrointestinal complaints. In CYC group, cessation of therapy was not needed however, two patients had gastro-intestinal complaints, nause/ vomiting, five had frequent infections that resulted in hospitalization and led to 2 weeks delay in regular dosing, three patients had cytopenias and three patients had elevated liver enzymes, which resulted in decreases in biweekly dosages. No treatment related death occurred during the study period..

\section{DISCUSSION}

Results of the presented study suggest that azathioprine is remarkably inferior to cyclophosphamide for the 
induction treatment of CTD-ILD, even in those with favorable baseline parameters, indicated by pulmonary function tests. Moreover, AZA was discontinued in almost $20 \%$ of patients due to intolerance. Percent improvement in FVC and HRCT reticulation were also more remarkable in CYC group. Thus, our results suggest that CYC might be the drug of choice as first line treatment in CTD-ILD, especially in those with SSc, RA and pSS. In IMS and IPAF patients, results of both regimens did not differ significantly, therefore AZA could be an alternative for CYC regimen.

There are few studies reported the use of AZA for induction therapy in SSc associated ILD [11, 12, 24], however, none of them compared AZA with intravenous cyclophosphamide and none included radiological assessments. . In these studies, changes in FVC percentages were conflicting, although there was a tendency for improvement in favor of oral CYC treatment. Results of our study are compatible with previous reports oral CYC treatment in SSc patients [25].

Literature on use of immunosuppressives in CTD-ILD is largely limited to SSc and data is scarce about use of them in other CTDs. Therefore treatment strategies are mainly based on extrapolation from the SSc trials. For RA associated ILD, there is only one study which compared AZA with MMF as initial treatment regimen[10]. In a large CTD-ILD cohort AZA showed marginal efficacy in stabilization of lung function, but subgroup analysis were not performed. In another CTD-ILD study, MMF demonstrated improvement of lung function, compared to placebo [26]. Comparative efficacy of CYC and MMF in RA-ILD was studied in a CTD-ILD cohort, and both showed similar improvements in lung functions [9]. Considering our results and available literature, CYC and MMF might be better options for the treatment of RA-ILD, compared to AZA for induction therapy. Efficacy of combined use of biologic agents with AZA, CYC and MMF are conflicting and not clear yet.

The EULAR/ACR recently up dated pSS management recommendations[27]. For pSS associated ILD, they recommended to use AZA for second line and CYC for rescue treatments, due to lack of randomized trials. - There is no prospective head to head study evaluating efficacy of drugs and current evidence for use of AZA in pSS-ILD is limited to case series [13, 28, 29]. None of studies reported radiographic severity or GAP index. In our study, progression was more frequent in AZA group,and FVC levels tend to decrease in AZA whereas tend to improve in CYC group (data not shown). Therefore, we recommend the use of CYC for pSS associated ILD, as in SSC and RA.

The management of inflammatory myositis associated ILD was challenging due to the risk of rapid deterioration of lung disease. AZA and CYC were compared in a recent meta-analysis[30]. In agreement with our results, both treatment modalities had similar efficacy on ILD in patients with IMS.

The interstitial lung disease with autoimmune features is a new concept and since the classification criteria was released in 2015, [31] data are rapidly growing on management of IPAF, although optimal treatment keep its uncertainty. In our cohort, induction therapy with AZA and CYC t had similar progression rates. However, the baseline radiographic extent of lung disease was more prominent in CYC group. Therefore, CYC might be a better choice in extensive disease.

Safety data related to AZA and CYC treatment were consistent with the literature. Treatment discontinuation rate related to AZA was $28.4 \%$ in our study, which is similar to previously reported $28 \%$ in a CTD-ILD study [10], Safety data of CYC is also compatible with current literature [32]. No fatality occurred during six months observation period in our study.

We have some limitations in our study. Although we have a reliable cohort registry, our study is retrospective and therefore choice of induction was based on the opinion of the physician on duty. However, number of patients were enough to show a clear difference between AZA and CYC in overall group and somewhat in some subgroups. Regarding the scarce data on literature, our study offers a valuable contribution to management of CTD-ILD in daily practice.

In conclusion, our results suggest that induction therapy with azathioprine might be an alternative option in IPAF patients with limited disease extent and inflammatory myositis associated ILD. However, CYC is 
superior to AZA in SSc, RA and pSS associated ILD and could be preferred over AZA treatment.

Acknowledgments : None

Funding: None.

Conflict of interest: The authors have declared no conflicts of interest

\section{References}

1. Kocheril, S.V., et al., Comparison of disease progression and mortality of connective tissue disease-related interstitial lung disease and idiopathic interstitial pneumonia. Arthritis Care \& Research: Official Journal of the American College of Rheumatology, 2005.53 (4): p. 549-557.

2. Steen, V.D. and T.A. Medsger, Changes in causes of death in systemic sclerosis, 1972-2002. Annals of the rheumatic diseases, 2007.66 (7): p. 940-944.

3. Dekhuijzen, P.N., et al., Corticosteroid treatment and nutritional deprivation cause a different pattern of atrophy in rat diaphragm. J Appl Physiol (1985), 1995. 78 (2): p. 629-37.

4. Fischer, A. and R. Du Bois, Interstitial lung disease in connective tissue disorders. The Lancet, 2012. 380 (9842): p. 689-698.

5. Maltzman, J.S. and G.A. Koretzky, Azathioprine: old drug, new actions. The Journal of clinical investigation, 2003. 111 (8): p. 1122-1124.

6. Strauss, G., W. Osen, and K.M. DEBATIN, Induction of apoptosis and modulation of activation and effector function in $T$ cells by immunosuppressive drugs. Clinical \& Experimental Immunology, 2002.128 (2): p. $255-266$.

7. Hall, A. and M. Tilby, Mechanisms of action of, and modes of resistance to, alkylating agents used in the treatment of haematological malignancies. Blood reviews, 1992. 6 (3): p. 163-173.

8. Tashkin, D.P., et al., Mycophenolate mofetil versus oral cyclophosphamide in scleroderma-related interstitial lung disease (SLS II): a randomised controlled, double-blind, parallel group trial.Lancet Respir Med, 2016. 4 (9): p. 708-719.

9. Barnes, H., et al., Cyclophosphamide for connective tissue disease-associated interstitial lung disease. Cochrane Database Syst Rev, 2018. 1 (1): p. Cd010908.

10. Oldham, J.M., et al., Azathioprine response in patients with fibrotic connective tissue disease-associated interstitial lung disease. Respir Med, 2016. 121 : p. 117-122.

11. Dheda, K., et al., Experience with azathioprine in systemic sclerosis associated with interstitial lung disease. Clinical rheumatology, 2004. 23 (4): p. 306-309.

12. Poormoghim, H., et al., Systemic sclerosis: comparison of efficacy of oral cyclophosphamide and azathioprine on skin score and pulmonary involvement-a retrospective study. Rheumatology international, 2014. 34 (12): p. 1691-1699.

13. Deheinzelin, D., et al., Interstitial lung disease in primary Sjogren's syndrome. Clinical-pathological evaluation and response to treatment. American journal of respiratory and critical care medicine, 1996. 154 (3): p. 794-799.

14. Van Den Hoogen, F., et al., 2013 classification criteria for systemic sclerosis: an American College of Rheumatology/European League against Rheumatism collaborative initiative. Arthritis \& Rheumatism, 2013. 65 (11): p. 2737-2747.

15. Kay, J. and K.S. Upchurch, ACR/EULAR 2010 rheumatoid arthritis classification criteria. Rheumatology, 2012. 51 (suppl_6): p. vi5-vi9. 
16. Shiboski, C.H., et al., 2016 American College of Rheumatology/European League Against Rheumatism classification criteria for primary Sjogren's syndrome: a consensus and data-driven methodology involving three international patient cohorts. Annals of the rheumatic diseases, 2017. 76 (1): p. 9-16.

17. Lundberg, I.E., et al., 2017 European League Against Rheumatism/American College of Rheumatology classification criteria for adult and juvenile idiopathic inflammatory myopathies and their major subgroups. Arthritis \& Rheumatology, 2017. 69 (12): p. 2271-2282.

18. Fischer, A., et al., "ERS/ATS Task Force onUndifferentiated Forms of CTD-ILD". An official European RespiratorySociety/American Thoracic Society research statement: interstitial pneumonia withautoimmunefeatures. 2015.

19. Nishiyama, O., et al., A simple assessment of dyspnoea as a prognostic indicator in idiopathic pulmonary fibrosis. Eur Respir J, 2010. 36 (5): p. 1067-72.

20. Society, A.T., Single-breath carbon monoxide diffusing capacity (transfer factor). Recommendations for a standard technique-1995 update. Am J Respir Crit Care Med, 1995. 152 : p. 185-198.

21. Goh, N.S., et al., Interstitial lung disease in systemic sclerosis: a simple staging system. American journal of respiratory and critical care medicine, 2008. 177 (11): p. 1248-1254.

22. Carter, J.D., et al., An analysis of MRI and ultrasound imaging in patients with gout who have normal plain radiographs.Rheumatology, 2009. 48 (11): p. 1442-1446.

23. Ryerson, C.J., et al., Predicting survival across chronic interstitial lung disease: the ILD-GAP model. Chest, 2014.145 (4): p. 723-728.

24. Nadashkevich, O., et al., A randomized unblinded trial of cyclophosphamide versus azathioprine in the treatment of systemic sclerosis. Clinical rheumatology, 2006. 25 (2): p. 205-212.

25. Tashkin, D.P., et al., Cyclophosphamide versus placebo in scleroderma lung disease. The New England journal of medicine, 2006.354 (25): p. 2655-2666.

26. Fischer, A., et al., Mycophenolate mofetil improves lung function in connective tissue disease-associated interstitial lung disease. The Journal of rheumatology, 2013. 40 (5): p. 640-646.

27. Brito-Zeron, P., et al., Efficacy and safety of topical and systemic medications: a systematic literature review informing the EULAR recommendations for the management of Sjogren's syndrome. RMD open, 2019. 5 (2): p. e001064-e001064.

28. Parambil, J.G., et al., Interstitial lung disease in primary Sjogren syndrome. Chest, 2006. 130 (5): p. 1489-1495.

29. Roca, F., et al., Interstitial lung disease in primary Sjogren's syndrome. Autoimmunity reviews, 2017. 16 (1): p. $48-54$.

30. Barba, T., et al., Treatment of idiopathic inflammatory myositis associated interstitial lung disease: A systematic review and meta-analysis. Autoimmunity Reviews, 2019. 18 (2): p. 113-122.

31. Fischer, A., et al., An official European Respiratory Society/American Thoracic Society research statement: interstitial pneumonia with autoimmune features. The European respiratory journal, 2015. 46 (4): p. $976-987$.

32. Cottin, V. and K.K. Brown, Interstitial lung disease associated with systemic sclerosis (SSc-ILD). Respiratory research, 2019. 20 (1): p. 13-13.

Table 1. Demographic and clinical features of azathioprine and cyclophosphamide groups.

\begin{tabular}{llll}
\hline & AZA group $(\mathrm{n}=43)$ & CYC group $(\mathrm{n}=72)$ & $\mathrm{p}$ \\
\hline Female, $\mathrm{n}(\%)$ & $39(90.7)$ & $57(77.9)$ & 0.126
\end{tabular}




\begin{tabular}{|c|c|c|c|}
\hline & AZA group $(\mathrm{n}=43)$ & CYC group $(\mathrm{n}=72)$ & $\mathrm{p}$ \\
\hline Age & $59.4 \pm 14.5$ & $53.8 \pm 14.5$ & 0.037 \\
\hline Smoking history, n (\%) & $6(14)$ & $15(20.8)$ & 0.629 \\
\hline $\begin{array}{l}\text { CTD disease duration } \\
\text { (median) }\end{array}$ & $6(1-16)$ & $6(1-12)$ & 0.719 \\
\hline $\begin{array}{l}\text { Concomitant steroid } \\
\text { usage** }\end{array}$ & $17(39.5)$ & $26(36)$ & 0.722 \\
\hline CTD subtype n (\%) SSc & $5(10.6) 8(50) 7(43.8) 9$ & $42(89.4) 8(50) 9(56.3)$ & $<0.001$ \\
\hline RA pSS IMS IPAF Other & (65) $13(65) 1(50)$ & $5(35) 7(35) 1(50)$ & \\
\hline $\begin{array}{l}\text { csDMARD usage n }(\%) \\
\text { bDMARD usage } \mathrm{n}(\%)\end{array}$ & $25(58) 1(2)$ & $45(62.5) 2(2.5)$ & 0.7710 .902 \\
\hline ILD pattern on HRCT, & $30(69.8) 4(9.5) 3(50)$ & $57(79) 38(90.5) 3(50)$ & $0.563[2] 0.552$ \\
\hline $\begin{array}{l}\mathrm{n}(\%) \text { NSIP n }(\%) S S c \\
R A p S S I M S I P A F\end{array}$ & $\begin{array}{l}5(45.5) 8(61) 10 \\
(66.7) 12(27.9) 1(20) 5\end{array}$ & $\begin{array}{l}6(54.5) 5(38.5) 5 \\
(33.3) 14(19.4) 4(80) 5\end{array}$ & \\
\hline $\begin{array}{l}\text { UIP n }(\%) S S c R A p S S \\
I M S I P A F\end{array}$ & $\begin{array}{l}(50) 2(40) 1(100) 3 \\
(60)\end{array}$ & $(50) 3(60)-2(40)$ & \\
\hline $\begin{array}{l}\text { Percentage of lung } \\
\text { reticulation on HRCT, } \\
\text { median (IQR) }\end{array}$ & $30(5-55)$ & $30(10-20)$ & 0.901 \\
\hline $\begin{array}{l}\text { Extent of Lung } \\
\text { Involvement (limited } \\
\text { group \%) }\end{array}$ & $16(37.2 \%)$ & $15(20.8 \%)$ & 0.081 \\
\hline MMRC*, (median) & $2(0-4)$ & $2(1-3)$ & 0.427 \\
\hline $\operatorname{DLCO}(\%) *($ median $)$ & $58(41-75)$ & $59(40-78)$ & 0.630 \\
\hline FVC, liter* & $2.33(1.37-3.29)$ & $2.19(1.13-3.25)$ & 0.689 \\
\hline $\begin{array}{l}\text { FVC, predicted \% } \\
\text { (median) }\end{array}$ & $83(55-111)$ & $72(61-83)$ & 0.032 \\
\hline GAP score & $1(0-2)$ & $1(0-2)$ & 0.33 \\
\hline
\end{tabular}

*Values were before the initiation of treatment, ${ }^{* *}$ none of the systemic sclerosis patients received this regimen. steroid dosage was defined as intravenous infusion of $250 \mathrm{mg}$ methylprednisone /bi-weekly . AZA: azathioprine, CYC: cyclophosphamide, CTD: connective tissue disease,bDMARD: Biologic disease modifiying anti rheumatismal drug, csDMARD: Sentetic disease modifiying anti rheumatismal druDLCO: diffusion carbon monoxide, FVC: forced vital capacity, GAP: Gender-Age-Physiology Score, IMS: Inflammatory myositis syndromes(Dermatomyositis/ Polymyositis/Anti-synthetase Syndrome)IPAF: Idiopathicinterstitial fibrosis with autoimmune features,MMRC: modified medical research council dyspnea scale, NSIP: non-specific interstitial pneumonia, OP: organizing pneumonia,pSS: primary Sjögren's syndrome, RA: Rheumatoid Arthritis, UIP: usual interstitial pneumonia,SSc: Systemic Sclerosis

Table 2. Treatment features and responses at six months

\begin{tabular}{|c|c|c|c|}
\hline & AZA group $(\mathrm{n}=43)$ & CYC group $(\mathrm{n}=72)$ & $\mathrm{p}$ \\
\hline$\overline{\mathrm{AZA} \text { dose, } \mathrm{mg} / \text { day }}$ & $101.78 \pm 32.22$ & na & \\
\hline $\begin{array}{l}\text { Total cumulative CYC } \\
\text { dose, mg }\end{array}$ & na & $8241 \pm 6190$ & \\
\hline $\begin{array}{l}\text { Combination of } \\
\text { medium-high dose } \\
\text { corticosteroids** }\end{array}$ & $65.1 \%$ & $51.4 \%$ & 0.176 \\
\hline$\Delta$ MMRC score & $0(-1$ to 1$)$ & $0(-1$ to 0$)$ & 0.031 \\
\hline$\Delta \mathrm{FCV}, \mathrm{ml}$ & $0.01(-0.27$ to 0.16$)$ & $0.06(-0.68$ to 1.5$)$ & 0.091 \\
\hline
\end{tabular}




\begin{tabular}{|c|c|c|c|}
\hline & AZA group $(n=43)$ & CYC group $(\mathrm{n}=72)$ & $\mathrm{p}$ \\
\hline$\Delta \mathrm{FCV}$, predicted $\%$ & $-1(-6$ to 3$)$ & $3(-2.75$ to 5$)$ & 0.025 \\
\hline $\begin{array}{l}\Delta \text { percent reticulation } \\
\text { on HRCT }\end{array}$ & $0(-0.62$ to 1.25$)$ & $0(-5$ to 0$)$ & 0.075 \\
\hline $\begin{array}{l}\text { Number of patients } \\
\text { with ILD progression }\end{array}$ & $17(39.5 \%)$ & $11(15.3 \%)$ & 0.013 \\
\hline 5 -year survival & $91 \%$ & $95 \%$ & 0.138 \\
\hline $\begin{array}{l}\text { Disease specific } \\
\text { evaluations, \% }\end{array}$ & $\begin{array}{l}\text { Disease specific } \\
\text { evaluations, \% }\end{array}$ & $\begin{array}{l}\text { Disease specific } \\
\text { evaluations, \% }\end{array}$ & $\begin{array}{l}\text { Disease specific } \\
\text { evaluations, \% }\end{array}$ \\
\hline$S S c(n=47)$ & 60 & $\begin{array}{l}11.9 \\
\text { progression }\end{array}$ & 0.029 \\
\hline $\mathrm{RA}(\mathrm{n}=16)$ & 62.5 & 25 & $>0.05$ \\
\hline $\mathrm{pSS}(\mathrm{n}=16)$ & 71.4 & 11.1 & 0.035 \\
\hline IMS $(n=14)$ & 11.1 & - & $>0.05$ \\
\hline $\operatorname{IPAF}(n=20)$ & 26.6 & 23.1 & $>0.05$ \\
\hline
\end{tabular}

*All values are presented as median (25-75 interquartile range) unless stated. AZA: azathioprineCTD: connective tissue disease, CYC: cyclophosphamide, FVC: forced vital capacity, GAP: Gender-Age-Physiology Score, IMS: Inflammatory myositis syndromes, IPAF: Idiopathic interstitial pneumonia with autoimmune features, MMRC: modified medical research council, pSS : primary Sjögren syndrome, RA: Rheumatoid Arthritis, SSc:Systemic Sclerosis.

Table 3. Safety and tolerability of drugs

\begin{tabular}{llll}
\hline & AZA $(\mathrm{n}=53)$ & CYC $(\mathrm{n}=72)$ & $\mathrm{p}$ \\
\hline Drug tolerability & $71.6 \%$ & $90.4 \%$ & $\mathbf{0 . 0 3 8}$ \\
Side effects n (\%) GIS & $8(18,6) 4(9) 2(4,5) 2$ & $14(19,4) 5(7) 3(4,2)-3$ & $\mathrm{p}>0.05$ \\
related** Elevated liver & $(4,5) 1(2,1)-$ & $(4,2) 5(7)$ & \\
enzymes Abdominal pain & & & \\
Cytopenia Frequent & & & \\
infection & & \\
\hline
\end{tabular}

Table 3: Safety analysis of treatment groups:* some patients developed multiple side effects. **GIS: Gastrointestinal system related side effects include nause and vomiting 